\title{
Analysis of surface degradation of high density polyethylene (HDPE) insulation material due to tracking
}

\author{
R SARATHI*, S CHANDRASEKAR, V SABARI GIRI, C VENKATASESHAIAH and \\ R VELMURUGAN ${ }^{\dagger}$ \\ Department of Electrical Engineering, ${ }^{\dagger}$ Composite Technology Centre, Indian Institute of Technology Madras, \\ Chennai 600 036, India
}

MS received 21 April 2003

\begin{abstract}
In the present work, tracking phenomena has been studied with HDPE material under a.c. voltage, with ammonium chloride as the contaminant. It is noticed that the tracking time depends on the conductivity and flow rate of the contaminant. The diffusion coefficient of the material was obtained. The thermal and chemical stability of the material were identified by carrying out a methodical experimental study. The physico-chemical analyses viz. wide angle X-ray diffraction (WAXD), differential scanning calorimetry (DSC) and dynamic mechanical analysis (DMA), were carried out and it was concluded that the mechanism of tracking process is due to the surface degradation. The surface condition of the insulation structure was characterized for any surface discharges or tracking, using the leakage current measurement, utilizing the wavelet concepts.
\end{abstract}

Keywords. Insulation; tracking; contact angle; WAXD; DSC; DMA; wavelet.

\section{Introduction}

Polymer materials are currently used as insulation structure in the power system network, because of their superior dielectric properties, light weight and compact, when compared to the porcelain or glass insulators (Gorur et al 1999). Based on the performance of the insulators, analysed by various means, it has been determined that most of the failure of outdoor insulation structure is the result of tracking, a peculiar phenomena that occurs on the surface of the insulation structure as a result of the creepage discharge thereby induced, all of which are attributed to the surface wetting and the degree of contamination. Once tracking occurs the surface electrical insulation property is lost completely and never recovers. To improve the reliability and the performance of insulation material, the tracking phenomena is being investigated worldwide (Gorur et al 1992; Alghamdi et al 1996; Kumagai and Yoshimura 2002).

Polyethylene is widely used in cable insulation and as an outer jacket to all self-supporting (ADSS) optical fibre cables worldwide (Kaidano 2000). The uptake of water in the bulk of the insulation wrapping the cable is affected by the hydrophobicity of the exterior surface. The presence of water in insulation leads to the formation of water/ electrical trees and eventual failure of cables. The unaged surface of the polyethylene material is usually hydropho-

\footnotetext{
*Author for correspondence
}

bic and therefore, resists the formation of a continuous film of water on the surface. Under wet or humid conditions and in the presence of pollutants, a gradual loss of hydrophobicity occurs, which is accelerated at high temperatures. The formation of a continuous film of water on the surface results in the formation of leakage current followed by dryband arcing and tracking on the surface, and thereby most of the optical fibre cable envelop gets damaged because of tracking (Kaidano 2000). Hence there is a need for reducing the damage of insulation material that results from tracking.

Having this in view, in the present work, tracking studies were carried out according to IEC-587 standard, with ammonium chloride as the contaminant, in high density polyethylene material, aged at different conditions. The influence of tracking resulting from conductivity of the contaminant and by the flow rate of the contaminant was thoroughly investigated in the present study. The surface condition of the aged insulation structures was characterized using contact angle and by the surface roughness measurement. The mechanical properties of the material were understood using the tensile test, impact test and flexural test. In addition, certain physico-chemical analyses, viz. wide angle X-ray diffraction (WAXD), differential scanning calorimetry and dynamic mechanical analysis (DMA), were all carried out to understand the tracking process in the insulation structure.

To diagnose the inception of tracking in the insulation structure, analysis of the leakage current provides some information. In the present work, utilizing the signal 
processing techniques, especially the wavelet technique, the leakage current signals were processed, which provide finger print identification of the surface condition of the insulators and the point of transition from surface discharge to tracking. In addition, the basic aspects of wavelets, the methodology adopted for characterization and selection of type of wavelets were also discussed in detail.

\section{Experimental}

In the present work, the tracking test was carried out following the IEC-587 test method, which is adopted for a.c. voltage application. The material used in the study was commercial grade HDPE material. The gap distance between the high voltage and the ground electrode was adjusted to be equal to $50 \mathrm{~mm}$. A schematic diagram of the experimental set up and the electrode configuration used in the present work are shown in figure $1 . \mathrm{NH}_{4} \mathrm{Cl}$ was used as the contaminant. The different flow rates of the contaminants were obtained using the control of the peristallitic pump. The a.c. voltage of $4.5 \mathrm{kV}$ was connected to the top electrode and the bottom electrode was solidly grounded. The times to failure due to tracking were arrived at once the arc inception took place near the bottom electrode and crossed two third of the gap; otherwise the process lasted for $6 \mathrm{~h}$ and the experiment was terminated. The conductivity of the contaminant was measured using a Lutron CD-4302.

The investigations on tracking were carried out with samples aged under different conditions.

\subsection{Corona ageing}

The surface of the HDPE specimen was placed in the corona zone for $24 \mathrm{~h}$ and then the samples were subjected to tracking. It was observed that the corona treated surface showed a milky white appearance. The temperature of the specimen at the time of termination of corona ageing was slightly above normal temperature.

\subsection{Thermal ageing}

The HDPE samples were placed in a temperature controlled oven maintained at $90^{\circ} \mathrm{C}$ for 15 days. The oven was circulated with clean air. The initial colour of the sample was white and upon termination of ageing, colour of the sample changed to yellowish orange.

\subsection{Cyclic thermal stress}

In this study, the HDPE samples were placed in temperature controlled oven maintained at $90^{\circ} \mathrm{C}$ for $8 \mathrm{~h}$ and on removal, it was suddenly quenched in distilled water bath and kept for $15 \mathrm{~min}$ to allow the surface temperature of the specimen to come back to normal room temperature. The cyclic process was continued for 25 times before carrying out experimental study.

\subsection{Water ageing}

The samples were immersed in a distilled water bath maintained at different temperatures for 15 days. In the present work, studies were carried out at room temperature, 60 and $90^{\circ} \mathrm{C}$. The samples were taken out and measured for increase in their weight at the required instant of time.

\subsection{Acid ageing}

The samples were immersed in oxalic acid, sulphuric acid, nitric acid and in the acid rain (of $\mathrm{pH}=2 \cdot 0$ ) for more than $250 \mathrm{~h}$. The $\mathrm{pH}$ value of the contaminant was measured using the digital $\mathrm{pH}$ meter model DI-707. The composition of acid rain is similar as mentioned in our earlier work (Sarathi et al 2002).

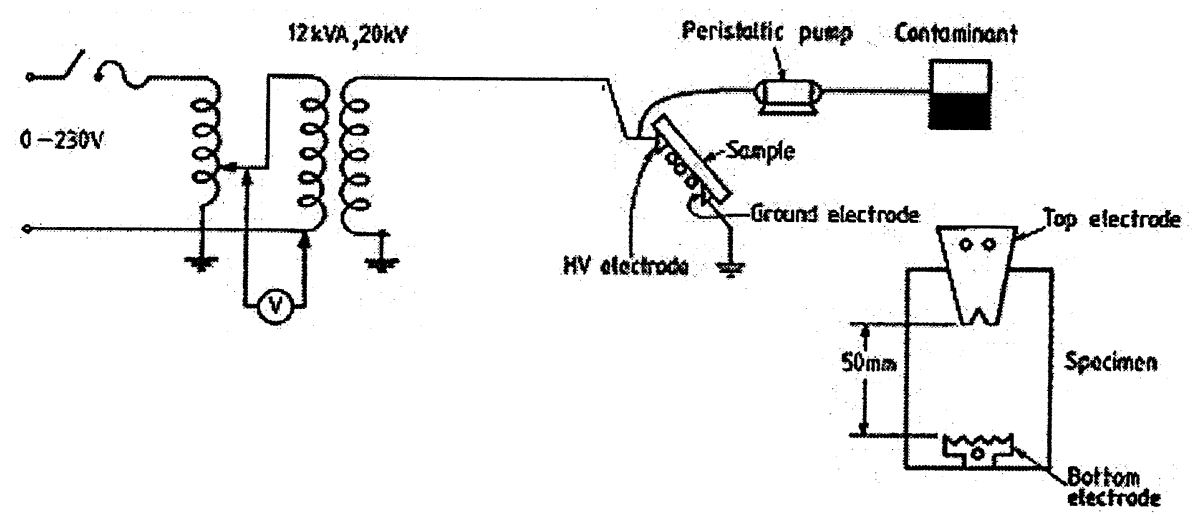

Figure 1. Experimental set up for tracking test (IEC-587). 


\subsection{Physico-chemical analysis}

2.6a Wide angle X-ray diffraction (WAXD): In the present work, WAXD measurements were done with Philips $\mathrm{X}$-ray diffractometer. A scan rate of $2 \% \mathrm{~min}$ at 2000 cycles using $\mathrm{CuK} \alpha$ radiation of wavelength $1.596 \AA$ was applied. A radial scan of Bragg angle (2 $\theta)$ vs intensity was obtained with an accuracy of $\pm 0.25^{\circ}$ at the location of the peak.

2.6b Differential scanning calorimetry (DSC): The melting behaviour of the specimens was observed using Perkin Elmer model DSC-2C apparatus. The experiments were performed in nitrogen atmosphere, at a heating rate of $10^{\circ} \mathrm{C} / \mathrm{min}$. Alumina was used as a standard.

\subsection{Tensile and flexural test}

Tensile test was carried out to understand the ability of a material to resist breaking under tensile stress. The breaking load and the elongation at break lengths were measured and the tensile stress was measured in the present work. In general, the ability of a material to resist deformation under a load is its flexural strength. For materials that do not break, the load at yield, typically measured at 5\% deformation/strain of the outer surface, is the flexural strength or flexural yield strength. By carrying out tensile test and flexural test, it is possible to understand the characteristics variation of the HDPE material used in the present work. The test was carried out in the universal testing machine of Instron 4301, $500 \mathrm{~kg}$ capacity. The pulling speed during the test was maintained at $5 \mathrm{~mm} / \mathrm{min}$.

\subsection{Impact test}

This test was carried out in the Frank model machine of 50J maximum capacity. Important factors which affect the toughness of a structure include low test temperatures, extra loading and high strain rates due to wind or impacts and the effect of stress concentrations such as notches and cracks. In the present work, the impact test was carried out to understand the material property due to aging of the material.

\subsection{Dynamic mechanic analysis (DMA)}

Visco-elastic measurements of both storage shear modulus and mechanical loss tangent were performed on TA instruments DMA-983 visco elastimeter using three-point bending method. Experiments were carried out over a wide frequency range $(2,5$ and $10 \mathrm{~Hz})$ in the temperature range $30-90^{\circ} \mathrm{C}$ under controlled sinusoidal strain, at a heating rate of $2^{\circ} \mathrm{C} / \mathrm{min}$ under a flow of nitrogen. All experiments were performed on a $20 \times 12 \mathrm{~mm}$ rectangular sheet at different frequencies. The visco-elastic properties, such as storage modulus $\left(E^{\prime}\right)$ and mechanical loss tangent $(\tan \delta)$, were recorded as a function of temperature and frequency. Dynamic mechanical analysis over a wide range of temperature and frequencies permits the determination of the viscoelastic behaviour of molten polymers and provides valuable insight into the relationship between structure, morphology and proportional properties of polymeric matrices.

\subsection{The tracking process under a.c. voltage}

The tracking process is basically a carbonaceous process. When high voltage is connected to the top electrode, the leakage current starts flowing in the conductive path formed by the contaminant and causes partial evaporation of the contaminant causing dry band in the gap. The dry band acts as a highly resistive zone in this process and the leakage current magnitude collapses to zero as shown in figure $2 \mathrm{a}$. The abrupt discontinuity in the leakage current is the indication for formation of the dry bands. The potential difference across the dry band increases causing arc formation. The continuous flow of the contaminant in the gap rewets the surface and the above process is repeated. The spark across the dry band results in slow increase in surface temperature and gradual rise in temperature causing carbonization/chain scission. The high temperatures of the arc in the gap as well as the high temperature due to possible reaction of $\mathrm{NH}_{4} \mathrm{Cl}$ with the insulation ma-
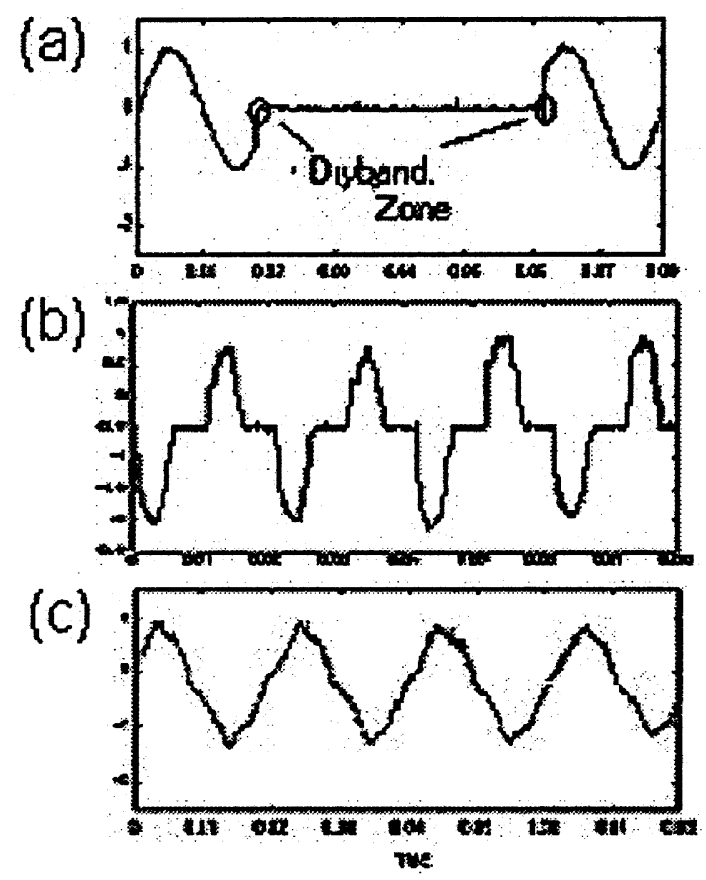

Figure 2. Typical leakage current waveforms: (a) current with dry band formation, (b) surface discharge current and (c) current during tracking. 
terial were considered to be responsible for the erosion of the material near the ground electrode, followed with tracking process. Sometimes, the arc burning across the gap is extinguished by the flow of contaminant and the entire process detailed above restarts. The localized bright spot occurring near the ground electrode causes high temperature rise leading to chemical change of the material forming carbonization of material near the spot resulting in localized erosion of the material. The variation in the shape of the current at the time of surface discharge and during tracking is shown in figures $2 \mathrm{~b}$ and $\mathrm{c}$, respectively.

However, an accumulation of the contaminants in the gap, over a period of time, permits more current to flow in the conductive path formed by the contaminant at the same applied voltage. Therefore, the entire process is accelerated so that heavy burning takes place between the electrodes causing carbonized path as shown in figure 3. This phase is identified as "failure due to tracking". The entire process progresses as shown in figure 4.

\subsection{Introduction to wavelets (Rioul 1991)}

The wavelet transform converts a signal from time domain to the time-scale domain. Wavelet analysis involves the breaking up of a signal into shifted and scaled versions of a single prototype function called the original or

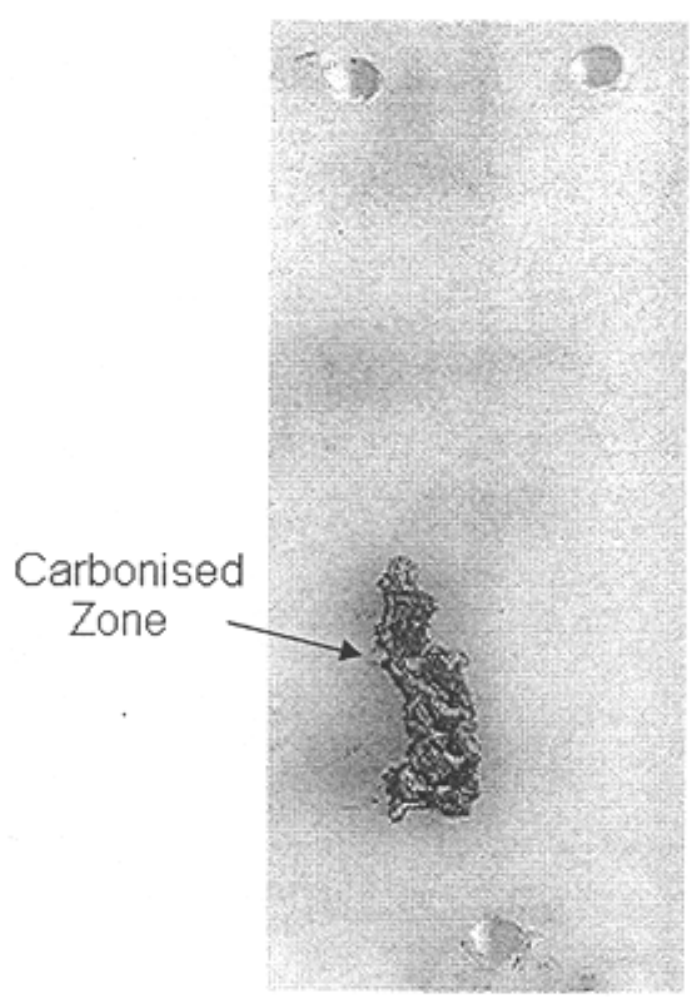

Figure 3. Tracking formed zone in HDPE material. mother wavelet. The Fourier transform gives us only the frequency domain representation of the signal. Therefore, it is suitable for analysing the signal where time-frequency resolution is needed. In the present work, it is essential to understand the cause of decomposition of the insulation structure and the instant of occurrence. Utilizing the inherent advantage of the wavelet analysis to the present work is important. A mathematical definition follows.

Let $x(t)$ be a signal defined in $L^{2}(R)$ space, which denotes a vector space for finite energy signals in a real continuous number system. Such signals satisfy

$$
\int_{-\infty}^{\infty} x^{2}(t) \mathrm{d} t<\infty
$$

The wavelet transform of $x(t)$ is then defined as

$$
\begin{aligned}
C W T_{x}^{\psi}(\tau, s) & =\psi_{x}^{\psi}(\tau, s) \\
& =\frac{1}{\sqrt{|s|}} \int x(t) \psi *\left(\frac{t-\tau}{s}\right) \mathrm{d} t,
\end{aligned}
$$

where $\psi(t)$ is called a base function (or mother wavelet). The asterisk denotes a complex conjugate, and $\tau, s \varepsilon R, s \neq 0$ are the dilation and translation parameters, respectively. The functions that are used as mother wavelets have zero net area. Instead of continuous dilation and translation, the mother wavelet may be dilated and translated discretely by selecting $s=a^{m}$ and $\tau=n b a^{m}$ where $a$ and $b$ are fixed constants with $a>1, b>0, m, n \varepsilon Z$ and $Z$ the set of positive integers. The discrete wavelet transform is given by

$$
D W T_{x}^{\psi}(m, n)=\frac{1}{\sqrt{a^{m}}} \int x(t) \psi *\left(\frac{t-n b a^{m}}{a^{m}}\right) \mathrm{d} t
$$

Let $c_{0}(n)$ be a discrete time signal recorded from a physical measuring device. The signal has to be decomposed into a detailed and smoothened representation. From the MSD technique, the decomposed signals at scale 1 are $c_{1}(n)$ and $d_{1}(n)$, where $c_{1}(n)$ is the low pass version of the original signal and $d_{1}(n)$ is the high pass representation of the original signal, $c_{0}(n)$, in the form of wavelet transform coefficients. They are defined as

$$
\begin{aligned}
& c_{1}(n)=\sum_{k} h(k-2 n) c_{0}(k), \\
& d_{1}(n)=\sum_{k} g(k-2 n) c_{0}(k),
\end{aligned}
$$

where $h(n)$ and $g(n)$ are the associated filter coefficients that decompose $c_{0}(n)$ into $c_{1}(n)$ and $d_{1}(n)$, respectively. 

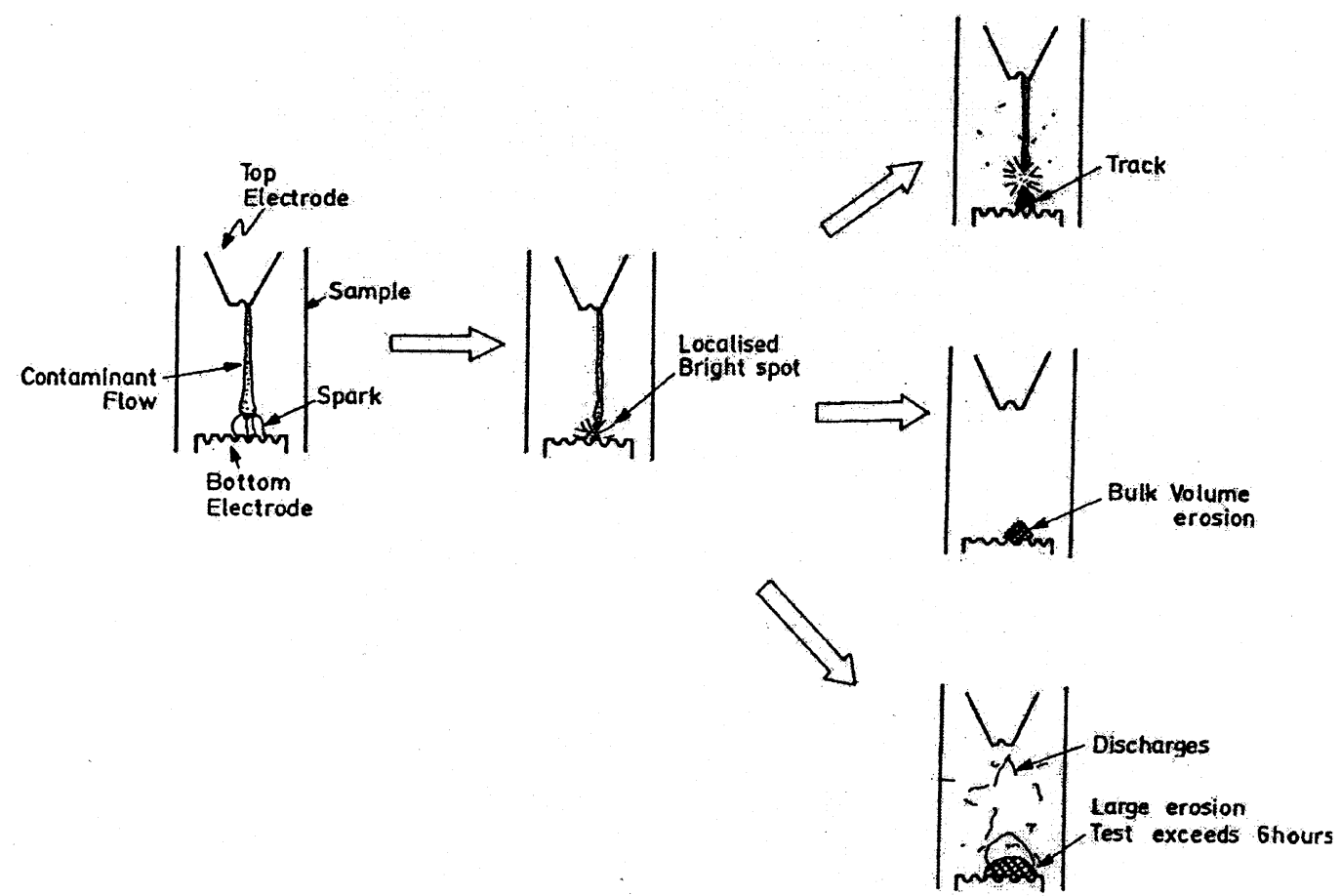

Figure 4. Schematic representation of tracking process.

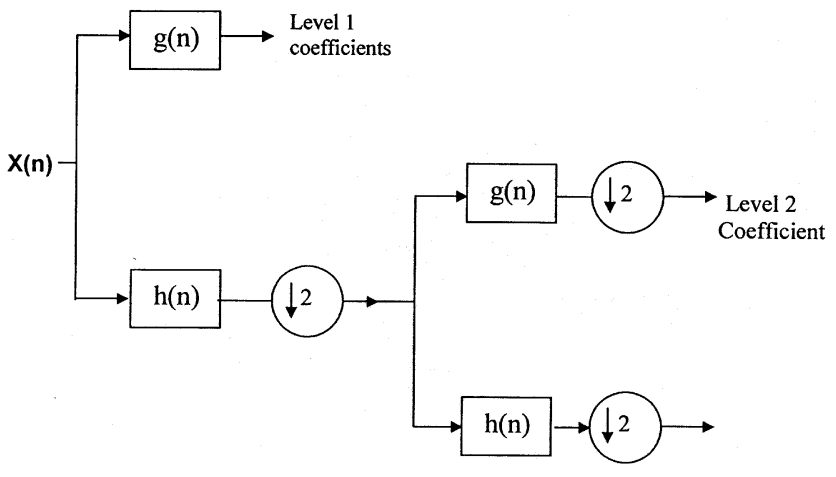

Figure 5. Schematic representation of implementation of multiresolution signal decomposition.

The next higher level decomposition is based on $c_{1}(n)$. The decomposition signal at scale 2 is given by

$$
\begin{aligned}
& c_{2}(n)=\sum_{k} h(k-2 n) c_{1}(k), \\
& d_{2}(n)=\sum_{k} g(k-2 n) c_{1}(k) .
\end{aligned}
$$

Higher scale decompositions are performed in the same way as described above. The implementation of the multiresolution signal decomposition technique is best described by figure 5 . Multiresolution signal decomposition is used to archive two important properties. The first is the localization property in time for any transient phenomena. This will appear by the presence of large coefficients at the time of disturbance. The second is the partitioning of the signal energy into different frequency bands. This gives an idea of the frequency content of the distorted signal. The standard deviation can be considered as a measure of the energy for a signal with zero mean. Therefore, the standard deviation at different resolution levels of the decomposed signal, standard multiresolution signal analysis (std_MRA) is used in the present work.

\section{Results and discussion}

Figure 6 shows the variation in the tracking time of the virgin HDPE material under different a.c. voltages with $\mathrm{NH}_{4} \mathrm{Cl}$ (conductivity of $2500 \mu \mathrm{S}$ ) as the contaminant. It is observed that an increase in the applied voltage shows a reduction in the tracking time of the insulation material. This indicates that the HDPE material is reliable for use as insulation for low voltage application where the electric field is less or otherwise when the applied electric field is less than $0.4 \mathrm{kV} / \mathrm{cm}$. Figure 7 shows the variations in tracking time of the virgin HDPE specimen under a.c. voltage with $\mathrm{NH}_{4} \mathrm{Cl}$ as the contaminant, at different conductivity levels. It is observed that an increase in conductivity of the contaminant reduces the tracking time. A similar characteristic behaviour was observed by Sarathi et al (2002) in EPDM specimen. The similar trends are 
also seen with the samples aged under different conditions viz. thermal aged specimen and cyclic thermal stress specimen. It is noticed that the cyclic aged specimen have tracking time much higher than the virgin specimen, whereas, the thermally aged specimen shows a reduction in the tracking time compared with the virgin specimen. The reason could be due to the variation in the property of the material due to ageing. The tracking time variation of the aged specimens is shown in table 1 . It is observed that the tracking time is drastically reduced for the samples aged in water. The cause for it is yet to be understood.

Figure 8 shows the variation in the tracking time of the HDPE specimen under the a.c. voltage with $\mathrm{NH}_{4} \mathrm{Cl}$ $(2500 \mu \mathrm{S})$ as contaminant, at different flow rates. It shows

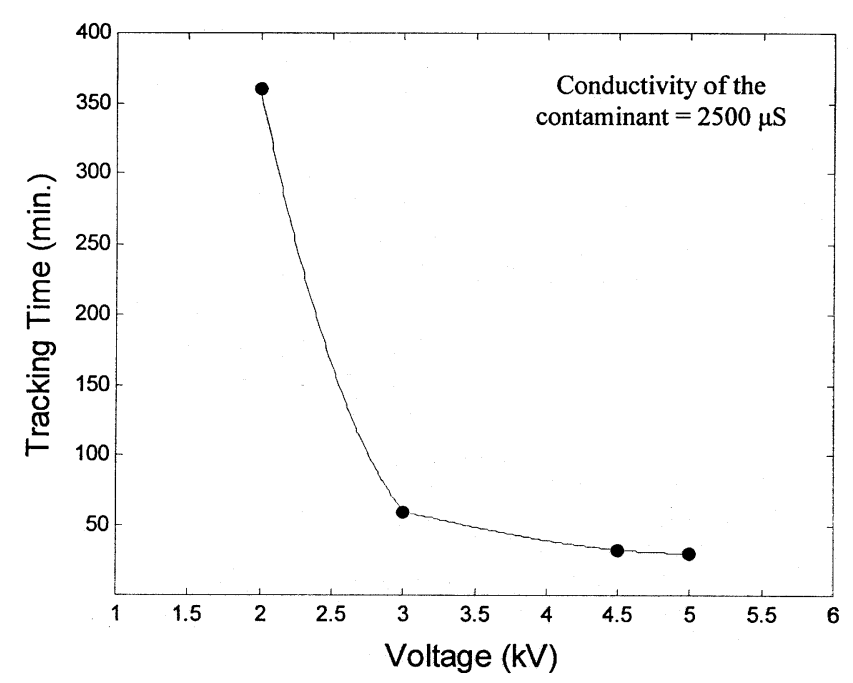

Figure 6. Variation in the tracking time of HDPE material at different voltage magnitudes.

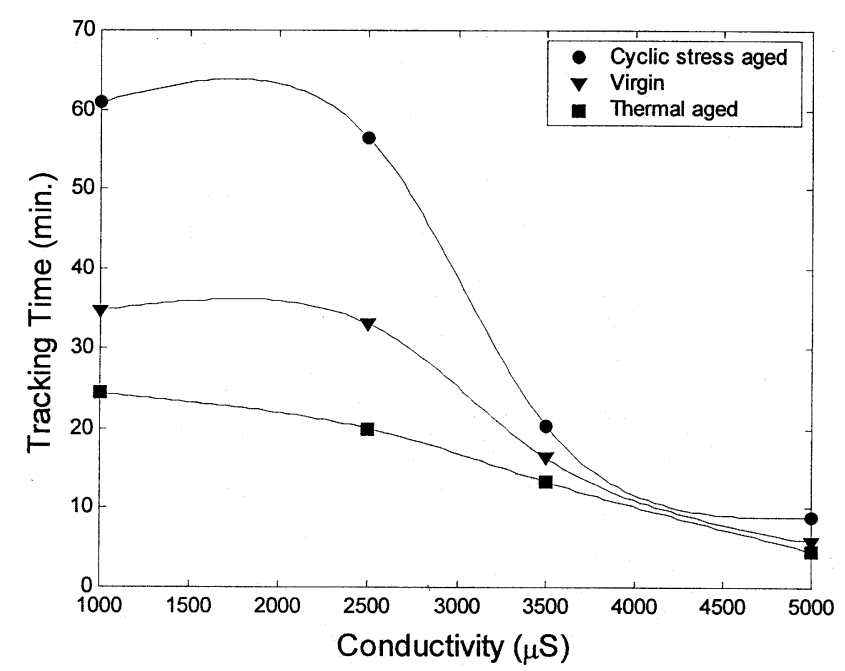

Figure 7. Variation in the tracking time of HDPE material at different conductivity levels. that increase in flow rate of the contaminant shows a reduction in the tracking time of the material. The reduction is very high with the cyclic aged specimen. This indicates that accumulation of contaminant over the surface increases the surface leakage current magnitude and increase power dissipation over the surface of the material causing temperature rise of the insulation material,

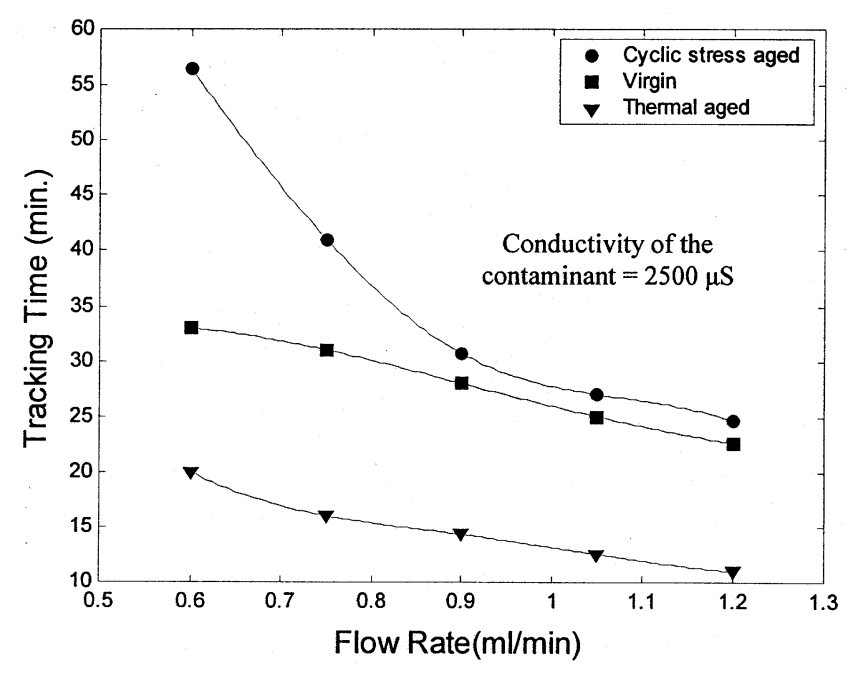

Figure 8. Variation in the tracking time of HDPE material at different flow rates of the contaminant.

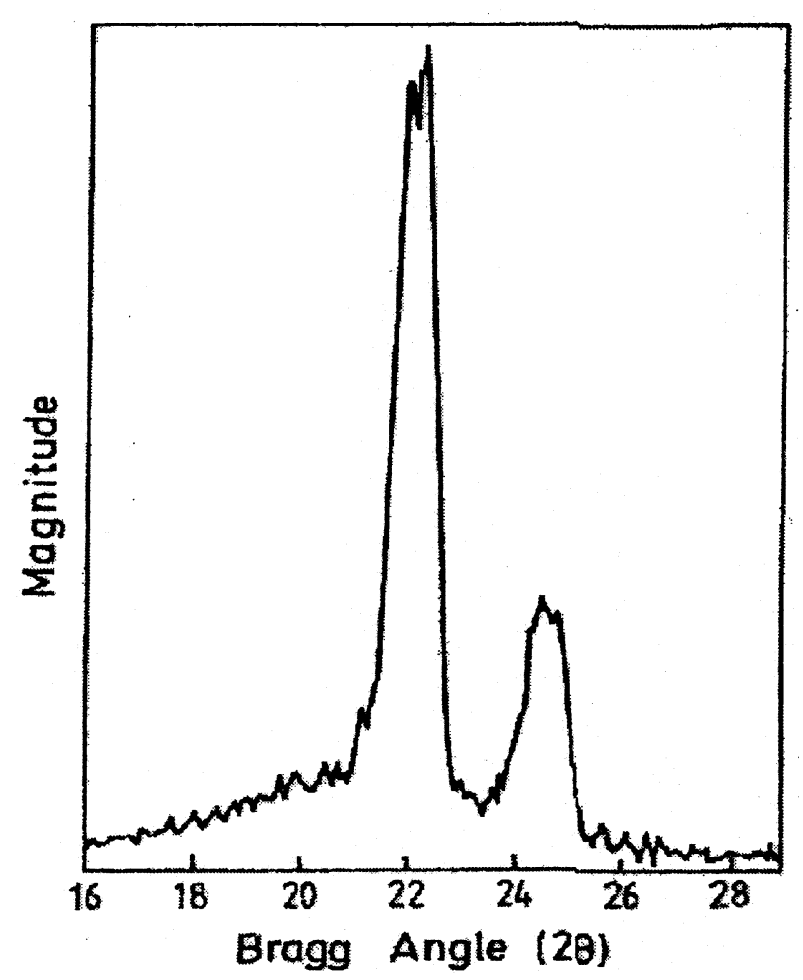

Figure 9. WAXD spectra of HDPE material. 
Table 1. Variation in the tracking time, contact angle and surface roughness of the HDPE material aged under different conditions.

\begin{tabular}{|c|c|c|c|c|}
\hline Sl. no. & Material & $\begin{array}{c}\text { Tracking } \\
\text { time } \\
(\mathrm{min})\end{array}$ & $\begin{array}{c}\text { Contact } \\
\text { angle } \\
(\theta)\end{array}$ & $\begin{array}{l}\text { Roughness } \\
\text { factor } \\
(\lambda)(\mu \mathrm{m})\end{array}$ \\
\hline 1 & Virgin & 33 & 85 & 1.89 \\
\hline 2 & Cyclic stress & 57 & 81 & $2 \cdot 20$ \\
\hline 3 & Thermal aged & 20 & 77 & $2 \cdot 41$ \\
\hline \multirow[t]{2}{*}{4} & Liquid nitrogen & & & \\
\hline & quenched & 30 & 72 & $2 \cdot 23$ \\
\hline 5 & Corona aged & 41 & 76 & $2 \cdot 60$ \\
\hline 6 & Water aged at $30^{\circ} \mathrm{C}$ & 12 & 60 & 1.93 \\
\hline 7 & Water aged at $60^{\circ} \mathrm{C}$ & 10 & 62 & $1 \cdot 89$ \\
\hline 8 & Water aged at $90^{\circ} \mathrm{C}$ & 16 & 66 & $2 \cdot 06$ \\
\hline 9 & $\mathrm{HNO}_{3}$ & 70 & 74 & $2 \cdot 46$ \\
\hline 10 & $\mathrm{H}_{2} \mathrm{SO}_{4}$ & 53 & 76 & $2 \cdot 43$ \\
\hline 11 & Oxalic acid & 53 & 80 & $2 \cdot 31$ \\
\hline 12 & Acid rain & 38 & 74 & $2 \cdot 24$ \\
\hline
\end{tabular}

thereby causing degradation of the material forming conducting path over the surface of the insulation material in a short time.

The diffusion coefficient of a saline solution into polyethylene material was determined at different temperatures. The samples were immersed in a distilled water bath maintained at different temperatures for 15 days. In the present work studies were carried out at room temperature, 60 and $90^{\circ} \mathrm{C}$. The samples were taken out and measured for increase in their weight at the required instant of time. Crank (1975) developed the relationship which indicates the level of saturation in absorption of liquid in the material. When diffusion is driven by the concentration gradient and if there is no chemical change between liquid and material, this would result in mass change and the rate of absorption will be initially linear with $t^{0.5}$, where ' $t$ ' is the time of absorption. Hence

$$
\frac{\Delta m(t)}{\Delta m_{\alpha}}=2 \sqrt{\frac{D t}{t^{2}}}\left\{\left[\sqrt{\frac{1}{\pi}}+2 \sum_{n=1}^{\infty}\left[(-1)^{n} \cdot \operatorname{ierf}\left(\frac{n 1}{2 \sqrt{D t}}\right)\right]\right]\right\}
$$

where $\Delta m(t)=m(t)-m(0)$ and $\Delta m_{\alpha}=m_{\alpha}-m(0)$. In this, $m(t)$ is mass at time 'tr'. $m(0)$ and $m(\alpha)$ are initial mass (at time $t=0$ ) and after infinite time, respectively. Crank (1975) provided the final equation to calculate diffusion coefficient and if the linear dependency in plot $\Delta m(t) /$ $\Delta m(\alpha)$ vs $t^{0.5}$ is not observed, then the diffusion constant is obtained by equating $\Delta m(t) / \Delta m_{\alpha}=0 \cdot 5$.

Simplifying the equation, we get

$$
D=\frac{\pi}{64} \frac{L_{0.5}^{2}}{t_{0.5}}
$$

Table 2. Variation in the diffusion coefficient of the material.

\begin{tabular}{lcc}
\hline Sl. no. & Type of material & Diffusion coefficient \\
\hline 1 & At $30^{\circ} \mathrm{C}$ & $12.06 \times 10^{-12}$ \\
2 & At $60^{\circ} \mathrm{C}$ & $23.04 \times 10^{-12}$ \\
3 & At $90^{\circ} \mathrm{C}$ & $33.20 \times 10^{-12}$ \\
\hline
\end{tabular}

Table 3. Variation in the crystallinity and the enthalpy of the HDPE material.

\begin{tabular}{llcc}
\hline S1. no. & \multicolumn{1}{c}{ Material } & Crystallinity & Enthalpy $(\mathrm{j} / \mathrm{g})$ \\
\hline 1 & Virgin & 44 & $179 \cdot 8$ \\
2 & Cyclic thermal aged & 63 & $243 \cdot 6$ \\
3 & Thermal aged & 51 & $189 \cdot 7$ \\
\hline
\end{tabular}

where $L$ is thickness of the specimen. In the present work the thickness of the material is $4 \mathrm{~mm}$. Table 2 shows the variation in the diffusion coefficient of the material aged in water at different temperatures. It is observed that at higher temperature the diffusion coefficient of the material increases.

The WAXD spectrum was obtained for a virgin specimen and for the tracking formed zone of the HDPE specimen. Because the WAXD patterns of the tracking formed zone are formally similar to the virgin samples, for brevity, only the virgin HDPE specimen spectrum is shown in figure 9. Agarwal and Tilley (1955) obtained the spectra of polyethylene showing peaks at 21.5 and 23.9 , which are characteristics of 110 and 220 lattice planes, respectively. Similar characteristics peak spectra were obtained in the present work. The main observation in the present work is that the WAXD obtained for the virgin and the surface tracked regions do not show any change in position of the peaks or their splitting of peaks throughout the scan range, which indicates that there is hardly any change (maximum of 5\%) of crystallinity and no new phases are introduced in the specimen. The WAXD of the aged specimens used in the present work have the same peak position except for change in the intensity of the peaks. It indicates that the ageing causes variation in the percentage of crystallinity of the material. In the present work, the percentage of crystallinity of the material was calculated using Hinrichsens method (Nath and Perlman 1989). Table 3 shows the change in crystallinity of the material aged under different conditions. It is observed that increase in crystallinity is observed with the cyclic thermal stress material than the continuous thermal stressed material. This confirms that the manufacturing process alters the characteristics of the material.

Figure 10 shows the DSC thermogram of the HDPE material. A similar melting characteristic with a shoulder peak at the rising part was observed for the specimen microtomed in the tracking formed zone. This change in 
characteristics of the material is due to change in the surface condition of the material due to tracking forming, causing free radicals, confirming that the tracking process as the degradation process.

The reaction kinetics responsible for the formation of free radicals is as follows and the process confirms that the tracking process as a degradation process.

$$
\mathrm{RH} \stackrel{\text { Homolytic cleavage }}{\longrightarrow} \underset{(\text { free radical) }}{\mathrm{R} *}+\mathrm{H}^{*}
$$

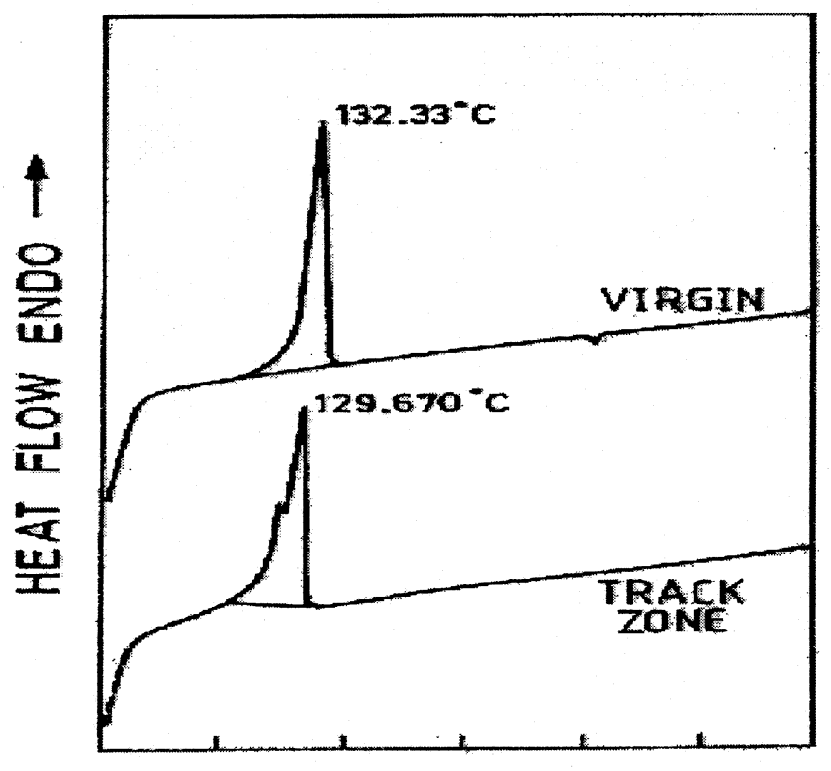

TEMPERATURE ( $\mathrm{C})$

Figure 10. DSC thermogram of HDPE material.

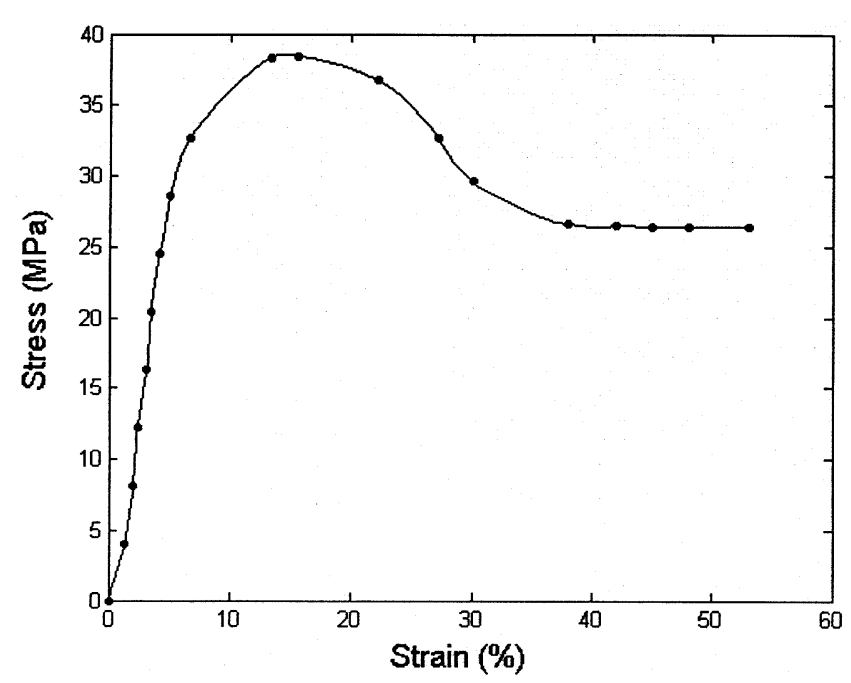

Figure 11. Tensile strength plot for HDPE specimen.
When free radical reacts with oxygen, an auto oxidation process starts. The free radical reacts with oxygen to form hydroperoxide. The unstable hydroperoxide decomposes and reproduces the free radical. The repetition of the process breaks the polymer chain.

$$
\begin{aligned}
& \mathrm{R}^{*}+\mathrm{O}_{2} \stackrel{\text { Oxidation }}{\longrightarrow} \mathrm{ROO}^{*} \text { (alkyl peroxy radical), } \\
& \mathrm{ROO} *+\mathrm{RH} \rightarrow \mathrm{ROOH}+\mathrm{R}^{*} \text { (free radical), } \\
& \mathrm{ROOH} \rightarrow \mathrm{RO}^{*}+\mathrm{OH} .
\end{aligned}
$$

Figure 11 shows the tensile strength plot of the virgin HDPE specimen. It is observed that the tensile strength of the virgin material is $39 \mathrm{MPa}$. Tensile strength were also measured for thermally aged specimen and for cyclic stress aged specimen as $37 \mathrm{MPa}$ and $36 \mathrm{MPa}$, respectively. This indicates that characteristic variation in the material property of the material is due to aging. To understand the intricate detail of the specimen, the impact test and flexural test were carried out. The results of flexural test and impact test were shown in tables 4 and 5, respectively. It is observed from the impact test that the cyclic stress ageing absorbs more energy compared to the virgin specimen. This indicates that the cyclic stress causes toughening of the material. The flexural test results indicate that the cyclic stress specimen show high displacement value compared to the virgin and the thermally aged material in the flexural test. It could be concluded easily from the results of impact test, flexural test and the tracking time of the aged material, that the materials with high toughness/stiffness causes increase in tracking time of the material.

Figure 12a shows the variation in storage modulus of HDPE specimen at different temperatures, measured at different frequencies. It is observed that as the frequency increases, the tensile storage modulus increases. Similar

Table 4. Flexural test result.

\begin{tabular}{llcc}
\hline S1. no. & \multicolumn{1}{c}{ Material } & $\begin{array}{c}\text { Peak load } \\
(\mathrm{kg})\end{array}$ & $\begin{array}{c}\text { Displacement } \\
(\mathrm{mm})\end{array}$ \\
\hline 1 & Virgin & $3 \cdot 6$ & $9 \cdot 67$ \\
2 & Cyclic thermal aged & $3 \cdot 4$ & $10 \cdot 5$ \\
3 & Thermally aged & $3 \cdot 8$ & $9 \cdot 74$ \\
\hline
\end{tabular}

Table 5. Impact test result.

\begin{tabular}{llc}
\hline S1. no. & \multicolumn{1}{c}{ Material } & Energy absorbed $(\mathrm{J})$ \\
\hline 1 & Virgin & 1.85 \\
2 & Cyclic thermal aged & 2.05 \\
3 & Thermally aged & 1.5 \\
\hline
\end{tabular}



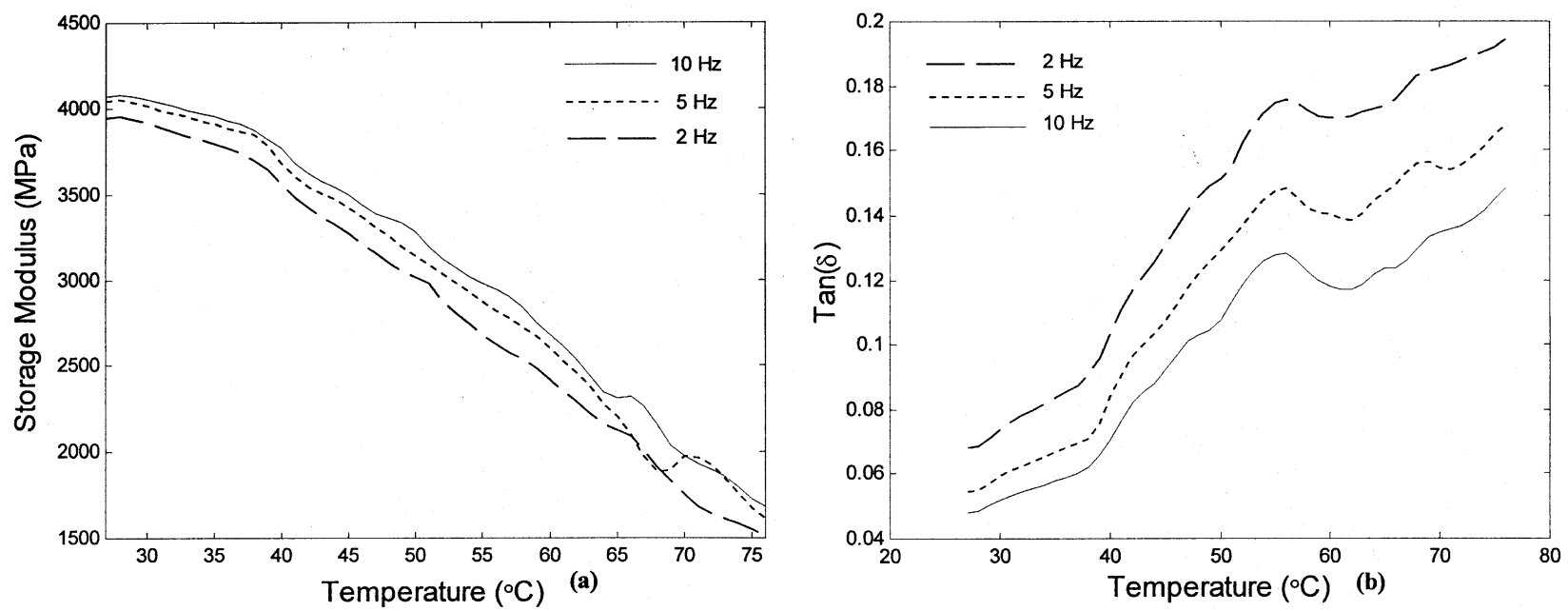

Figure 12. (a) Plot of storage modulus of HDPE material as a function of temperature and frequency and $(\mathbf{b})$ plot of $\tan (\delta)$ as function of temperature and frequency.
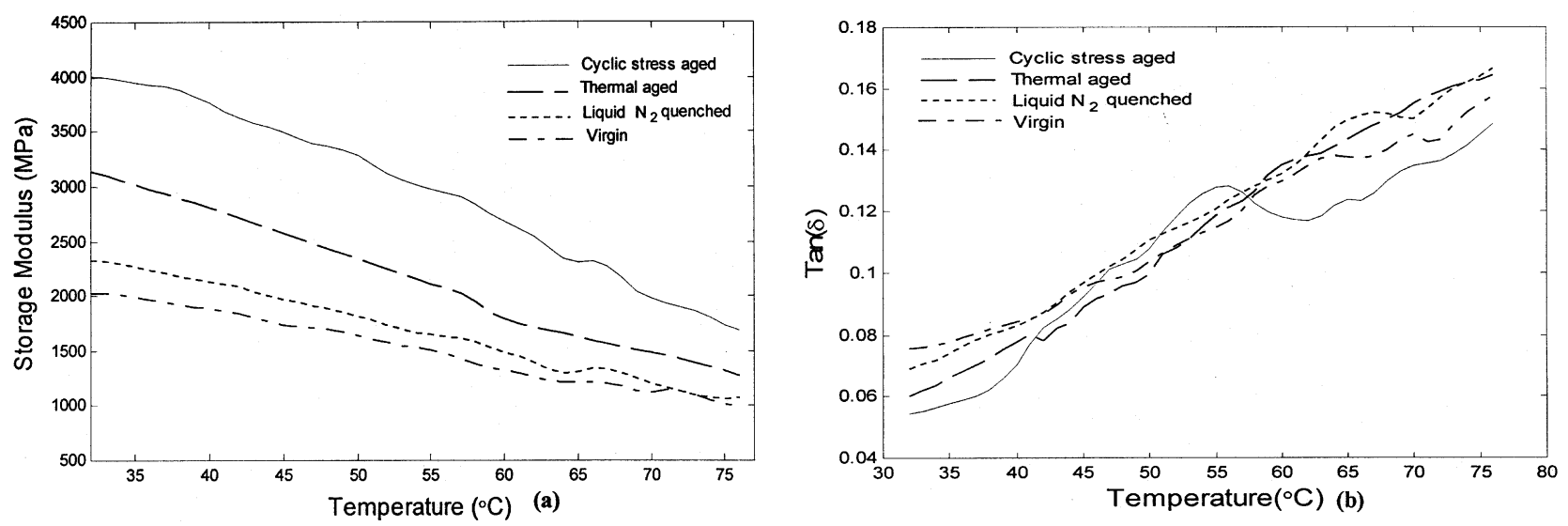

Figure 13. (a) Plot of storage modulus of HDPE material as a function of temperature and frequency and (b) plot of $\tan (\delta)$ as function of temperature and frequency.

observation was noticed with the polypropylene material (Lopez-Manchado and Arroyo 2000). Figure 12b shows the variation in the $\tan (\delta)$, which is a dimensionless parameter and conveys no physical magnitude, but is a measure of the ratio of energy lost to the energy stored in a cyclic deformation, is the loss tangent, which is less at higher frequencies, in the range studied. Figure 13 shows the variation in the storage modulus of the insulation material aged under different conditions, measured at one frequency. The loss factor of the material is nearly the same in all materials aged under different conditions. It is observed that the storage modulus is high for the sample treated for cyclic stress compared to the virgin material. It could be due to the increase in crystallinity of the material, causing increase in the packing density of the material. A similar characteristic was observed with the liquid nitrogen quenched specimens. These two materials have high intensity of the crystalline peak compared to the virgin material, measured through the WAXD studies. While understanding the tracking behaviour of the insulation material, it is observed that the cyclic stress aged specimen have higher tracking time compared to the virgin material. It indicates that a material with high storage modulus shows higher tracking time. This impact test results are in conformity with the DMA test results to conclude that the material with high toughness/stiffness improves the tracking time of the polymeric material.

It is essential to understand the level of leakage current magnitude and the frequency contents in the leakage current to know the surface condition of the insulators. It is well known that FFT analysis could provide sufficient information about the harmonic contents present in the 

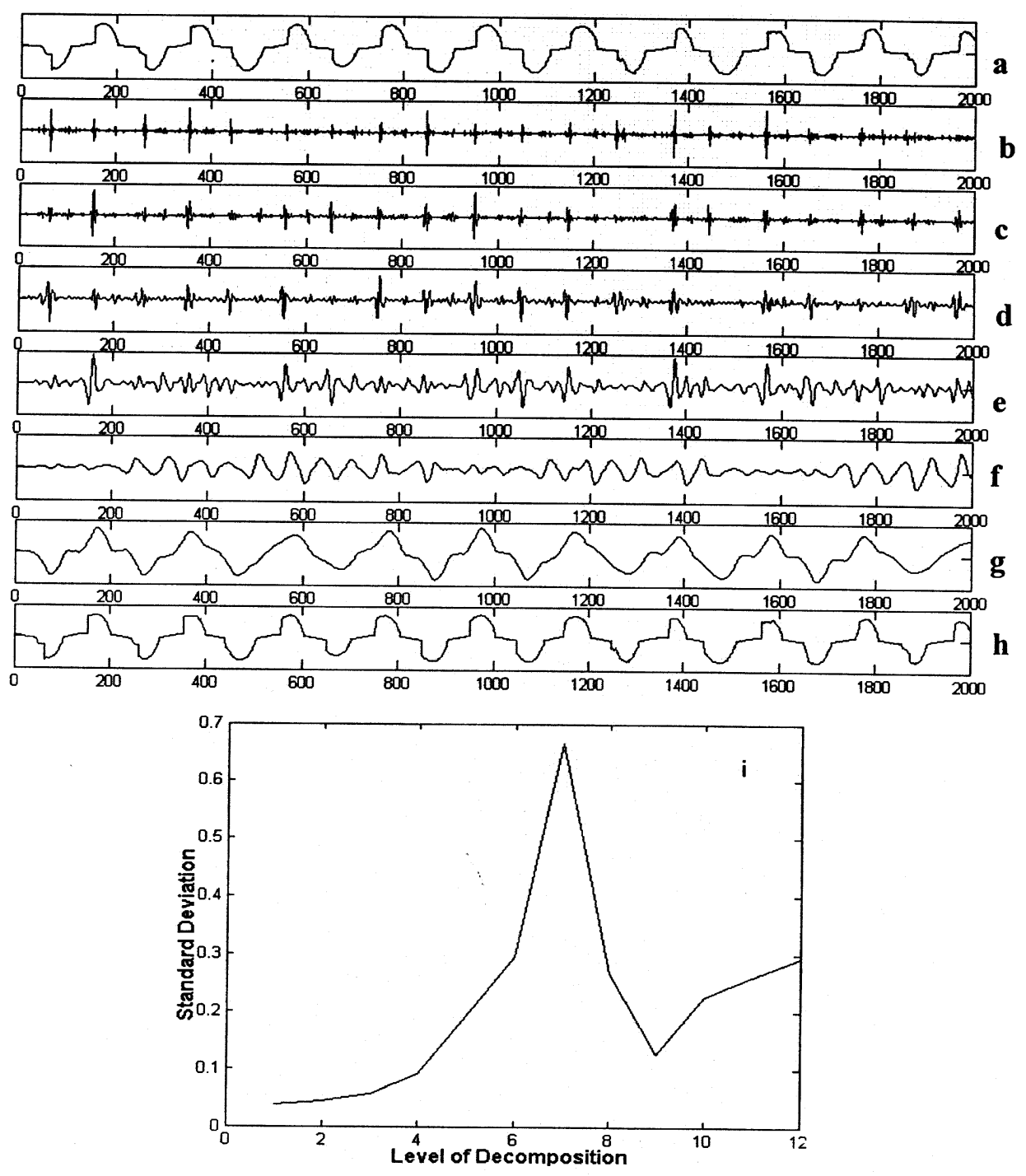

Figure 14. a. Surface discharge waveform, b-f. detailed coefficients, g. final approximation, h. reconstructed signal and i. std_MRA plot.

signal. For non-stationary waves the reconstruction of the original signal from the fast fourier transformed signal is highly questionable (Gaouda et al 1999). Suda (2001) studied the leakage current in actual insulators and identified higher harmonic contents in the signal generated and classified different stages from the point of inception of discharge to the point of flashover. The results of the present work in the polyethylene flat plate samples are on similar lines. It is well known that Daub 4 wavelet and Daub 6 wavelet are very useful in identifying any transition in the signal due to high frequencies. In the present work Daub4 wavelet is used. Figure 14 shows the leakage current waveform and five levels of decomposition of the signal. The horizontal axis represents time (in samples with a sampling frequency of $10 \mathrm{kHz}$ ) and the vertical axis represents magnitude. Figure 14a shows the leakage current waveform and figures $14 \mathrm{~b}-\mathrm{g}$ show the five-level decomposition of the signal. The reconstructed signal waveform is shown in figure $14 \mathrm{~h}$. The standard deviation multiresolution curve is shown in figure 14i. Characteristic variation in the std_MRA plot is observed and it provides finger print identification for classification. A typical leakage current waveform and the std_MRA curves of normal current, surface discharges and at the time of tracking are shown in figure 15. One predominant peak is observed for the normal sinusoidal current. High and low frequency contents are noticed in the surface discharge signals. In addition, at the time of tracking, the std_MRA results indicate predominant changes in values at all levels compared to the surface discharge/normal leakage current signals. These classification rules are so simple for anyone to identify the operating condition of 


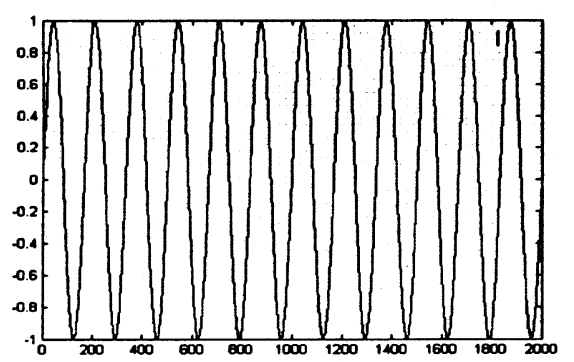

(a)
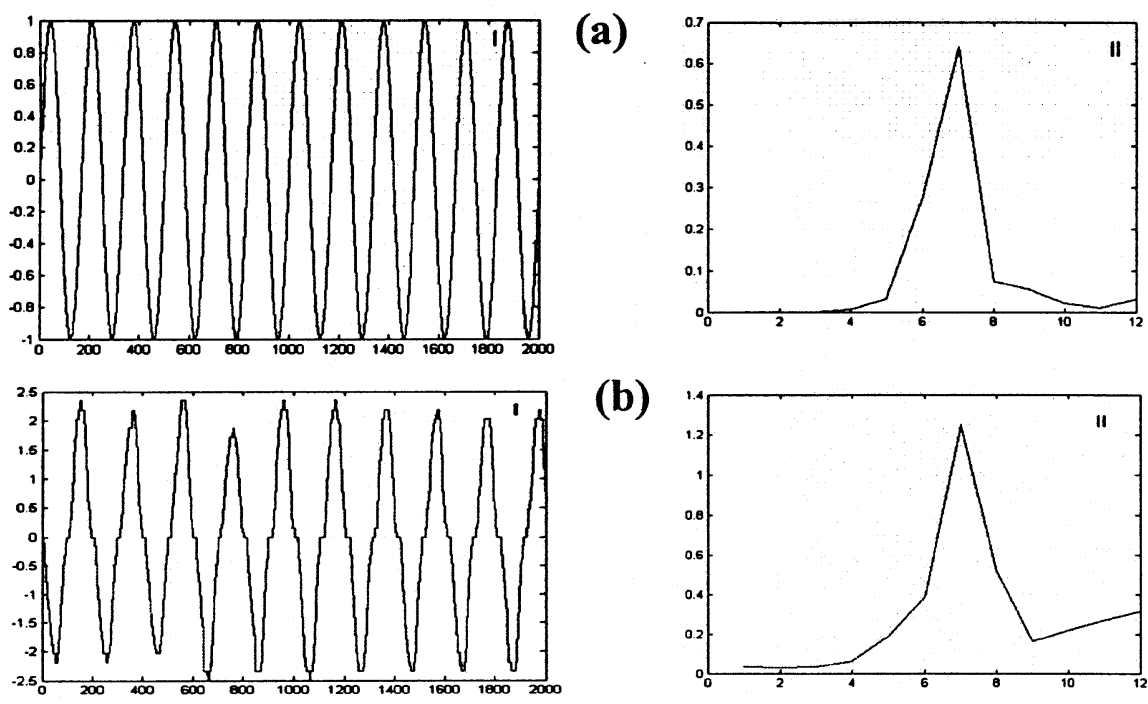

(b)
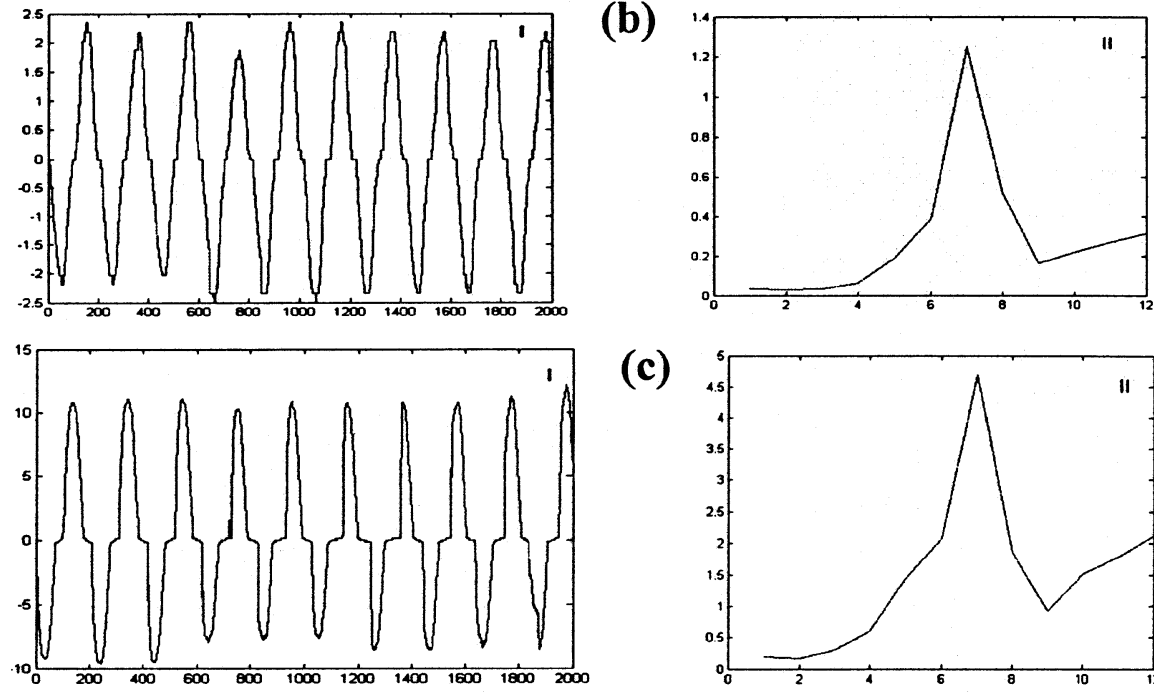

(c)

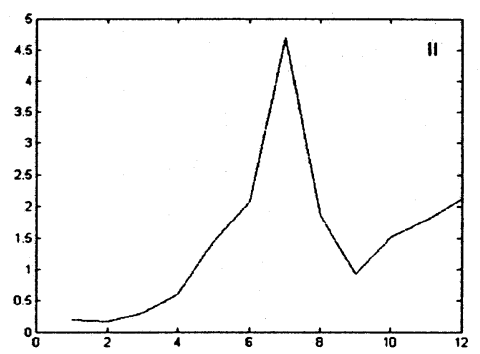

Figure 15. Typical std_MRA plots: (a) pure sinusoid, (b) surface discharge and (c) tracking.

the insulators. The std_MRA curves show different patterns and help one to identify the surface condition of the insulation structure.

\section{Conclusions}

It is observed that HDPE is an ideal outdoor insulation structure for low voltage applications. An increase in conductivity and flow rate of the contaminant exhibited a reduction in the tracking time of the insulation material. It is confirmed that the material properties significantly alter the tracking time of the insulation structure. The contact angle and the surface roughness of the material varies irrespective of the type of ageing. The diffusion coefficient of the samples increases with the temperature of the water bath.

The WAXD and DSC studies indicate no addition of new phases in the insulation structure due to ageing process. A variation in percentage of crystallinity of the material is noted with the thermally aged and the cyclic aged specimens. A reduction in the enthalpy of the material in the tracking formed zone is observed from the DSC results. This indicates that only the surface damage has occurred in the insulation structure. The mechanisms of degradation process which occurred in the material were explained. The tensile strength results indicate that aging of the material alters the mechanical property of the material. The impact and flexural test indicates that the material with high toughness/stiffness causes increase in the tracking time of the material.

The DMA analysis indicates that the storage moduli of the material increases with increase in frequency. The variation in the storage modulus of the material with ageing of the material was observed. The loss tangent of the material is high at low frequencies, irrespective of the type of aging of the material.

The standard multi-resolution signal analysis curve provides finger print identification of deviation of leakage current from normal sinusoid with the addition of harmonic content. The magnitude of high and low frequency contents increase when surface discharge occurs. Characteristic increase in values at all points is observed in the standard MRA curve with the tracking current.

\section{Acknowledgement}

One of the authors (RS) wishes to thank Prof. L Satish, Indian Institute of Science, Bangalore, for his kind suggestions on wavelets. 


\section{References}

Agarwal S L and Tilley G P 1955 J. Appl. Polym. Sci. 1817 Alghamdi A S G, Aukland D W, Risino A J and Varlow B R 1996 IEEE Trans. Dielectr. \& Electr. Insul. 3439

Crank J 1975 Mathematics of diffusion (Oxford: Clarendon) 2nd edn

Gaouda A M et al 1999 IEEE Trans. Power Delivery 141469

Gorur R S, Karady G G, Agota A, Shah M and Yates A M 1992 IEEE Trans. Power Delivery 7525

Gorur R S, Cherney E A and Burnham J T 1999 Outdoor insulators (Phoenix, Arizona: Ravi S Gorur Inc.)

IEC Publication 5871984 Testing method for evaluating the resistance of tracking and erosion of electrical insulating materials used under severe ambient conditions

Kaidano F, Munteanu R and Sheinfain G 2000 IEEE Electr. Insul. Mag. 1616

Kumagai S and Yoshimura N 2001 IEEE Trans. Dielectr. \& Electr. Insul. 8203

Lopez-Manchado M A and Arroyo M 2000 Polymer 417761

Nath R and Perlman M M 1989 IEEE Trans. Electr. Insul. E124409

Rioul O and Vitterli M 1991 IEEE Signal Process. Mag. 814

Sarathy R, Rao U M and Venkataseshaiah C 2002 Polym. Test. 21463

Suda T 2001 IEEE Trans. Dielectr. \& Electr. Insul. 8705 